\title{
Decade-long trends in the timeliness of receipt of a primary percutaneous coronary intervention
}

This article was published in the following Dove Press journal:

Clinical Epidemiology

6 June 2016

Number of times this article has been viewed

\author{
Han-Yang Chen' \\ Joel M Gore',2 \\ Kate L Lapane ${ }^{\prime}$ \\ Jorge Yarzebski' \\ Sharina D Person' \\ Catarina I Kiefe' \\ Robert J Goldberg ${ }^{1,3}$ \\ 'Department of Quantitative Health \\ Sciences, ${ }^{2}$ Department of Medicine, \\ ${ }^{3}$ Meyers Primary Care Institute, \\ University of Massachusetts Medical \\ School, Worcester, MA, USA
}

Correspondence: Robert J Goldberg Division of Epidemiology of Chronic Diseases and Vulnerable Populations, Department of Quantitative Health Sciences, University of Massachusetts Medical School, 368 Plantation Street,

Worcester, MA 01605, USA

$\mathrm{Tel}+|\mathbf{5 0 8} 856399|$

Fax + I 5088568993

Email Robert.Goldberg@umassmed.edu
Objectives: The purpose of this study was to examine decade-long trends (2001-2011) in, and factors associated with, door-to-balloon time within 90 minutes of hospital presentation among patients hospitalized with ST-segment elevation myocardial infarction (STEMI) who received a primary percutaneous coronary intervention (PCI).

Methods: Residents of central Massachusetts hospitalized with STEMI who received a primary PCI at two major PCI-capable medical centers in central Massachusetts on a biennial basis between 2001 and 2011 comprised the study population $(n=629)$. Multivariable regression analyses were used to examine factors associated with failing to receive a primary PCI within 90 minutes after emergency department (ED) arrival.

Results: The average age of this patient population was 61.9 years; $30.5 \%$ were women, and $91.7 \%$ were White. During the years under study, $50.9 \%$ of patients received a primary PCI within 90 minutes of ED arrival; this proportion increased from 2001/2003 (17.2\%) to 2009/2011 $(70.5 \%)(P<0.001)$. Having previously undergone coronary artery bypass graft surgery, arriving at the ED by car/walk-in and during off-hours were significantly associated with a higher risk of failing to receive a primary PCI within 90 minutes of ED arrival.

Conclusion: The likelihood of receiving a timely primary PCI in residents of central Massachusetts hospitalized with STEMI at the major teaching/community medical centers increased dramatically during the years under study. Several groups were identified for purposes of heightened surveillance and intervention efforts to reduce the likelihood of failing to receive a timely primary PCI among patients acutely diagnosed with STEMI.

Keywords: epidemiology, ST-segment elevation myocardial infarction, percutaneous coronary intervention

\section{Introduction}

The prompt administration of coronary reperfusion therapy for patients with an evolving acute myocardial infarction (AMI) is crucial in reducing mortality and the risk of serious clinical complications in these patients. ${ }^{1}$ During the past decade, primary percutaneous coronary intervention (PCI) has gradually replaced thrombolysis as the main revascularization strategy for patients presenting with ST-segment elevation myocardial infarction (STEMI), since primary PCI has been found to be superior to thrombolytic therapy when performed rapidly by expert teams. ${ }^{2}$ Because the effectiveness of primary PCI may be limited by delays in its prompt delivery, current clinical guidelines have recommended a door-to-balloon time of 90 minutes or less for patients hospitalized with STEMI who undergo a primary PCI. ${ }^{1}$ 
To date, while a number of studies have described the timing of receipt of a PCI in patients presenting to the hospital with STEMI, there are little population-based data available describing contemporary trends in the magnitude of, and factors associated with, door-to-balloon times in patients experiencing STEMI who receive a primary PCI; ${ }^{3-6}$ the limited studies in this area have shown mixed results of improvement in door-to-balloon time during varying study years and an inconsistent profile of patients who fail to be treated within recommended guidelines. ${ }^{3-6}$ Inasmuch, there is a need to examine relatively contemporary long-term trends in the extent of, and potential risk factors associated with, delays in door-to-balloon time among patients hospitalized with STEMI who undergo a primary PCI, particularly from the more generalizable perspective of a population-based investigation.

The primary objective of our study was to describe decade-long (2001-2011) trends in the extent of delay from hospital emergency department (ED) presentation to initiation of primary PCI among patients hospitalized with STEMI. Our secondary objective was to examine factors associated with the failure to receive a primary PCI within 90 minutes after ED arrival among patients hospitalized with STEMI. Data from the Worcester Heart Attack Study were used for purposes of this investigation..$^{7-10}$

\section{Methods}

Described elsewhere in detail, ${ }^{7-10}$ the Worcester Heart Attack Study is an ongoing population-based investigation examining long-term trends in the descriptive epidemiology of AMI in residents of the Worcester, MA, metropolitan area (2000 census $=478,000$ ) hospitalized at all eleven medical centers in central Massachusetts on an approximate biennial basis between 1975 and 2011. ${ }^{7-10}$ We reviewed patient's medical records on an approximate biennial basis since the inception of this study due to the availability of federal funding support and design features of this observational study.

Computerized printouts of patients discharged from all greater Worcester hospitals with possible AMI (International Classification of Disease (ninth revision) codes: 410-414, 786.5) were identified. Cases of possible AMI were independently validated using predefined criteria for AMI; ${ }^{7-10}$ these criteria included a suggestive clinical history, increases in several serum biomarkers (eg, creatine kinase, creatine kinase-MB, and troponin values), and serial electrocardiographic findings during hospitalization consistent with the presence of AMI. Patients who satisfied at least two of these three criteria and who were residents of the Worcester metropolitan area since this study was population-based were included. A diagnosis of
STEMI was made when new ST-segment elevation was present at the J point in two or more contiguous leads. ${ }^{11}$

For the purposes of this study, we restricted our sample to adult residents of the Worcester metropolitan area who were hospitalized with STEMI and received a primary PCI at a PCI-capable hospital between 2001 and 2011, which were our most recent study years and allowed us to examine decade-long trends in the end points of interest. Among the eleven medical centers in the Worcester metropolitan area, the vast majority (99\%) of PCIs were performed at the two major urban teaching and community hospitals in the city of Worcester. Patients who received thrombolytic therapy during hospitalization were excluded since they did not meet the criteria for receiving a primary PCI. Door-to-balloon time was defined as the time interval from the patient's arrival at the hospital ED to inflation of the balloon to restore coronary flow. Patients who were transferred from another hospital were excluded, since the clinical guidelines of door-to-balloon time within 90 minutes were recommended for those who were initially seen at a PCI-capable hospital. To increase the likelihood that we were assessing patients who received a primary PCI, we excluded patients with hospital delay times that exceeded 6 hours $^{5,12}$ and those who did not have door-to-balloon times documented in their hospital medical records. This study used secondary data from the review of medical records of patients hospitalized with AMI. No patients were directly contacted and patient consent was not required. This study was approved by the Institutional Review Board at the University of Massachusetts Medical School.

\section{Data collection}

Trained nurses and physicians abstracted information on patients' demographic characteristics, medical history, clinical data, and treatment practices through the review of hospital medical records. Information on patient's sociodemographic characteristics (eg, age, sex, race, marital status), year of hospitalization, history of previously diagnosed comorbidities (eg, stroke, diabetes, heart failure), prior coronary revascularization (PCI or coronary artery bypass graft [CABG] surgery), AMI event order (initial vs prior), hospital ED arrival day and time, mode of transportation (car/walked-in vs ambulance), and door-to-balloon time was collected.

\section{Data analysis}

To increase the available sample size, and for ease of analysis and interpretation, we aggregated the six individual 
study years into three 2-year strata (2001/2003, earliest; 2005/2007, middle; and 2009/2011, most recent) for purposes of examining trends in our principal study outcomes. Door-to-balloon time was further dichotomized as $\leq 90 \mathrm{~min}$ utes versus $>90$ minutes based on current clinical guidelines recommendations. ${ }^{1}$ Differences in the distribution of patient demographic and clinical characteristics between patients hospitalized during the three aggregated time periods were examined using the analysis of variance test for continuous variables and the chi-square test for categorical variables. The Cochran-Armitage tests and linear regression models were used to test for linear trends over time among categorical and continuous variables, respectively.

Delays to the receipt of a primary PCI after hospital arrival were examined by calculating the mean and median door-to-balloon times and the proportion of patients who received a primary PCI within 90 minutes among patients hospitalized with STEMI during the years under study. Due to the relatively common nature of the primary study outcome (ie, $>10 \%$ ), and the advantage of providing relative risk estimates, multivariable-adjusted Poisson regression models with robust error variance ${ }^{13}$ were used to examine the association between the main explanatory variable of time period of hospitalization (2001/2003, earliest; 2005/2007, middle; and 2009/2011, most recent) and the outcome of whether patients failed to receive a primary PCI within 90 minutes after hospital ED arrival (ie, door-to-balloon time: $>90 \mathrm{~min}-$ utes vs $\leq 90$ minutes) while adjusting for several potentially confounding variables of prognostic importance. We dummy coded this variable with the earliest study years (2001/2003) serving as the reference group.

Several covariates associated with delay to the receipt of a primary PCI in patients hospitalized with STEMI in prior studies were examined. ${ }^{3,4,14,15}$ These factors included age, sex, race (White vs non-White), marital status (married vs unmarried), previously diagnosed comorbid conditions (angina, atrial fibrillation, heart failure, hypertension, peripheral vascular disease, stroke, diabetes, chronic obstructive pulmonary disease, depression, and chronic kidney disease), prior coronary revascularization (PCI or CABG surgery), AMI event order (initial vs prior), hospital ED arrival time (regular hours: 8 am-6 pm, weekday vs off-hours: before 8 am or after 6 pm, weekday and weekend), and mode of transportation (car/walked-in vs ambulance). We also repeated our multivariable-adjusted Poisson regression analysis using data from the first and the last 2-year time clusters for purposes of exploring the association between various demographic and clinical factors with delays in the receipt of a primary PCI.
The results of our Poisson regression models with robust error variance were presented as multivariable-adjusted risk ratios (RRs) with accompanying 95\% CIs. All statistical analyses were conducted using SAS Version 9.3 (SAS Institute, Inc., Cary, NC, USA).

\section{Results \\ Study population characteristics}

During the years under study, there were a total of 1,853 patients hospitalized with STEMI at our participating sites; among these patients, 1,224 patients received a PCI. For purposes of examining patients hospitalized with STEMI who received a primary PCI within the guideline-recommended door-to-balloon time, we sequentially excluded patients who received thrombolytic therapy during their acute hospitalization ( $\mathrm{n}=81)$, who were transferred from another hospital $(\mathrm{n}=325)$, whose door-to-balloon time exceeded 6 hours $(\mathrm{n}=150)$, and who did not have door-to-balloon times documented $(n=39)$. The final study population consisted of 629 adult residents of central Massachusetts who were hospitalized with STEMI and received a primary PCI at the two major PCI-capable urban teaching and community hospitals in the Worcester metropolitan area on a biennial basis between 2001 and 2011.

Overall, the average age of this patient population was 61.9 years; $30.5 \%$ were women, $91.7 \%$ were White, and $62.0 \%$ were married. In addition, $75.8 \%$ of our study sample was hospitalized for an initial AMI, $75.9 \%$ were transported to the study hospitals by ambulance, and $53.1 \%$ arrived at participating EDs during off-hours (Table 1).

During the most recent years under study, patients who were hospitalized with STEMI and received a primary PCI were more likely to have a history of peripheral vascular disease or have prior coronary revascularization, compared to those hospitalized with STEMI during earlier study periods (Table 1).

\section{Trends in door-to-balloon time}

The average delay time from the patient's arrival at the ED to inflation of the balloon to restore coronary flow during the years under study was 102 minutes. There was a marked decrease in the mean delay time from 2001/2003 (141 minutes) to 2009/2011 (85 minutes) (Figure 1). The median delay time from the patient's arrival at the hospital ED to balloon inflation during the years under study was 89 minutes. There was also a significant decrease in the median duration of PCIassociated delay from 2001/2003 (133 minutes) to 2009/2011 (73 minutes) (Figure 1). 
Table I Characteristics of patients who were hospitalized with STEMI and received a primary PCI: Worcester Heart Attack Study

\begin{tabular}{|c|c|c|c|c|c|}
\hline Characteristics & $\begin{array}{l}2001 / 2003 \\
(n=163)\end{array}$ & $\begin{array}{l}2005 / 2007 \\
(n=208)\end{array}$ & $\begin{array}{l}2009 / 20 I I \\
(n=258)\end{array}$ & $P$-value ${ }^{a}$ & $P$ for trend ${ }^{b}$ \\
\hline Age, mean, years & 61.8 & 62.8 & 61.3 & 0.45 & 0.68 \\
\hline \multicolumn{6}{|l|}{ Age, $\%$} \\
\hline$<55$ years & 33.1 & 29.1 & 33.9 & 0.83 & \\
\hline $55-64$ years & 27.6 & 28.1 & 27.6 & & \\
\hline $65-74$ years & 16.0 & 20.2 & 19.3 & & \\
\hline$\geq 75$ years & 23.3 & 22.7 & 19.3 & & \\
\hline Female, \% & 30.7 & 30.3 & 30.6 & 0.99 & 0.99 \\
\hline White, \% & 94.4 & 90.2 & 91.4 & 0.37 & 0.39 \\
\hline Married, \% & 64.6 & 61.7 & 60.5 & 0.70 & 0.42 \\
\hline Initial AMI, \% & 79.1 & 75.0 & 74.4 & $0.5 \mathrm{I}$ & 0.29 \\
\hline ED off-hours arrival, \% & 52.1 & 61.5 & 46.9 & 0.007 & 0.15 \\
\hline Transport to hospital by car/walked-in, \% & 18.7 & 23.6 & 27.6 & 0.12 & 0.040 \\
\hline \multicolumn{6}{|l|}{ Medical history, $\%$} \\
\hline Angina & 16.6 & 7.2 & 2.3 & $<0.001$ & $<0.001$ \\
\hline Atrial fibrillation & 4.3 & 3.8 & 3.5 & 0.92 & 0.67 \\
\hline Chronic kidney disease & 6.7 & 11.1 & 8.9 & 0.36 & 0.56 \\
\hline Chronic obstructive pulmonary disease & 11.0 & 9.1 & 10.9 & 0.78 & 0.98 \\
\hline Depression & 10.4 & 14.4 & 17.4 & 0.14 & 0.048 \\
\hline Diabetes & 28.8 & 18.3 & 26.7 & 0.035 & 0.88 \\
\hline Heart failure & 5.5 & 7.2 & 6.2 & 0.80 & 0.85 \\
\hline Hypertension & 63.2 & 56.7 & 65.5 & 0.14 & 0.47 \\
\hline Peripheral vascular disease & 4.3 & 15.9 & 12.8 & 0.002 & 0.02 \\
\hline Stroke & 8.0 & 2.9 & 1.6 & 0.002 & 0.001 \\
\hline Prior $\mathrm{PCl}$ & 15.3 & 21.6 & 27.5 & 0.013 & 0.003 \\
\hline Prior CABG surgery & 3.7 & 4.3 & 5.8 & 0.57 & 0.30 \\
\hline \multicolumn{6}{|l|}{ Physiological parameters on admission } \\
\hline Initial heart rate, mean, beats/min & 79.8 & 76.8 & 80.8 & 0.10 & 0.60 \\
\hline Systolic blood pressure, mean, $\mathrm{mmHg}$ & 133.4 & 137.8 & 137.8 & 0.27 & 0.14 \\
\hline Diastolic blood pressure, mean, $\mathrm{mmHg}$ & 78.3 & 79.8 & 81.6 & 0.25 & 0.10 \\
\hline Serum glucose, mean, $\mathrm{mg} / \mathrm{dL}$ & 173.1 & 158.2 & 166.8 & 0.12 & 0.37 \\
\hline Estimated glomerular filtration rate, mean, $\mathrm{mL} / \mathrm{min} / 1.73 \mathrm{~m}^{2}$ & 68.6 & 69.0 & 58.4 & $<0.0001$ & $<0.0001$ \\
\hline
\end{tabular}

Notes: ${ }^{a}$-values derived from ANOVA tests for continuous variables and chi-square tests for categorical variables. ${ }^{b}$-values derived from Cochran-Armitage tests for categorical variables and linear regression models for continuous variables.

Abbreviations: STEMI, ST-segment elevation myocardial infarction; PCI, percutaneous coronary intervention; AMI, acute myocardial infection; ED, emergency department; CABG, coronary artery bypass graft; min, minutes; ANOVA, analysis of variance.

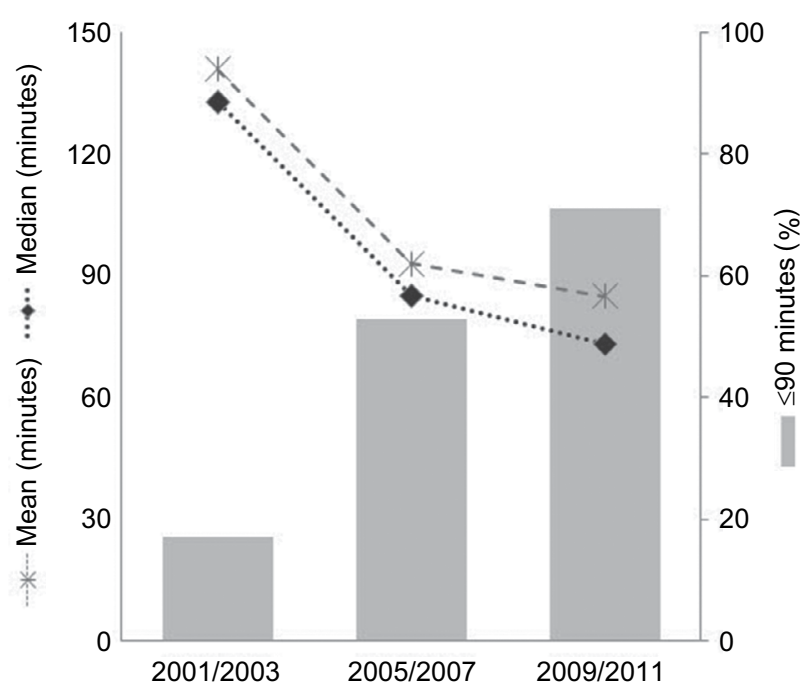

Figure I Trends in door-to-balloon time among patients hospitalized with STsegment elevation myocardial infarction: Worcester Heart Attack Study.
Among all study patients who underwent a primary PCI, 50.9\% of these patients received the intervention within 90 minutes of their arrival at the ED. There was a dramatic increase in the proportion of patients with STEMI who received a primary PCI within guidelinerecommended 90 minutes between 2001/2003 (17.2\%) and 2009/2011 (70.5\%) ( $P$ for trend $<0.001)$ (Figure 1$)$. Among all study patients who received a primary PCI within 90 minutes, $46.3 \%$ were treated during the first hour after arrival at the hospital ED; this proportion significantly increased from $10.7 \%$ in $2001 / 2003$ to $47.8 \%$ in $2009 / 2011(P<0.001)$.

In examining changing trends in the failure to receive a primary PCI within 90 minutes, after adjusting for several demographic characteristics and clinical factors, there was a significant reduction in the risk of failing to receive a primary PCI in $2005 / 2007(\mathrm{RR}=0.53,95 \% \mathrm{CI}=0.40-0.72)$ 
Table 2 Association between time period of hospitalization and failure to receive a primary $\mathrm{PCl}$ within 90 minutes among patients hospitalized with STEMI: Worcester Heart Attack Study

\begin{tabular}{llll}
\hline $\begin{array}{l}\text { Study } \\
\text { period }\end{array}$ & $\begin{array}{l}\text { Failure to } \\
\text { receive a } \\
\text { primary } \\
\text { PCl within } \\
\mathbf{9 0} \text { minutes }\end{array}$ & $\begin{array}{l}\text { Adjusted for } \\
\text { sociodemographics, } \\
\text { comorbidities, } \\
\text { prior coronary } \\
\text { revascularization }\end{array}$ & $\begin{array}{l}\text { Further } \\
\text { adjusted for } \\
\text { in-hospital } \\
\text { factors }^{\mathrm{b}}\end{array}$ \\
\cline { 2 - 4 } & Adjusted RR (95\% CI) \\
\hline $2001 / 2003$ & 82.8 & 1.00 & 1.00 \\
$2005 / 2007$ & 47.1 & $0.56(0.42-0.75)$ & $0.53(0.40-0.72)$ \\
$2009 / 2011$ & 29.5 & $0.36(0.26-0.49)$ & $0.36(0.26-0.50)$
\end{tabular}

Notes: aAjusted for sociodemographic characteristics, previously diagnosed comorbid conditions, and prior coronary revascularization (PCl or CABG surgery). ${ }^{b}$ Adjusted for sociodemographic characteristics, previously diagnosed comorbid conditions, prior coronary revascularization ( $\mathrm{PCl}$ or CABG surgery), $\mathrm{AMI}$ order, ED arrival time, and mode of transportation.

Abbreviations: $\mathrm{PCl}$, percutaneous coronary intervention; STEMI, ST-segment elevation myocardial infarction; RR, risk ratio; CABG, coronary artery bypass graft; $\mathrm{AMI}$, acute myocardial infarction; $\mathrm{ED}$, emergency department; $\mathrm{Cl}$, confidence interval.

and in $2009 / 2011(\mathrm{RR}=0.36,95 \% \mathrm{CI}=0.26-0.50) \mathrm{com}-$ pared with those hospitalized with STEMI in 2001/2003 (Table 2).

\section{Factors associated with failure to receive a primary $\mathrm{PCl}$ within 90 minutes}

Using multivariable-adjusted regression analyses, we examined the role of several prognostic factors associated with failure to receive a primary PCI within 90 minutes in all study patients (Table 2). Having previously undergone CABG surgery and arriving at the ED by car/walked-in and during off-hours were significantly associated with a higher risk of failing to receive a primary PCI within 90 minutes (Table 3 ). In addition, we further examined the role of various demographic and clinical factors with the failure to receive a primary PCI within 90 minutes using data from the first and the last 2-year time clusters (Table 4). Compared with the results in our overall study population, some similar and discrepant results were found in the time periods analyzed. In these subgroup analyses, arriving at the ED by car/walk-in during off-hours was no longer significantly associated with failing to receive a primary PCI within 90 minutes; however, having previously undergone CABG surgery was significantly associated with a higher risk of failing to receive a primary PCI within 90 minutes in the last 2-year time period.

\section{Discussion}

The results of this observational study suggest that, among greater Worcester residents who were hospitalized for STEMI and received a primary PCI at the two major PCI-capable hospitals in central Massachusetts between 2001 and 2011, there was a fourfold increase in the proportion of patients who received a primary PCI within guideline-recommended 90 minutes during the years under study. Having previously undergone CABG surgery and arriving at the ED by car/ walked-in and during off-hours were significantly associated with a higher likelihood of failing to receive a primary PCI within 90 minutes at participating study hospitals.

\section{Trends in, and magnitude of, door-to-balloon time}

Timely medical care is crucial to reducing mortality and the risk of serious clinical complications in patients experiencing signs and symptoms of an AMI. This is because it can maximize the benefits of evidence-based treatments and possibly reduce the likelihood of sudden cardiac deaths and the eventual size of the infarct. Although primary PCI has been shown to improve outcomes in patients with STEMI, its effectiveness may be limited by delays in its more timely delivery. ${ }^{1}$

Since 1999, clinical practice guidelines for the management of patients with STEMI have recommended door-toballoon times of 90 minutes or less. ${ }^{1,16,17}$ However, earlier data from the National Registry of Myocardial Infarction, which examined 33,647 patients hospitalized with STEMI who received a primary PCI between 1999 and 2002 at 421 US hospitals, reported that only $35 \%$ of patients were treated within the recommended 90 minutes after arrival at the hospital; meaningful improvements in door-to-balloon times over the study period were not observed. ${ }^{3}$

These discouraging findings led to several national efforts dedicated to reducing door-to-balloon time in patients hospitalized with STEMI. The Centers for Medicare and Medicaid Services and the Joint Commission began using door-to-balloon time as a performance measure in $2002 .{ }^{18} \mathrm{In}$ 2006, the Centers for Medicare and Medicaid Services began publicly reporting hospital achievement of door-to-balloon times of 90 minutes or less. In November 2006, the American College of Cardiology, the American Heart Association, and several other organizations launched the "Door-to-Balloon (D2B): An Alliance for Quality" campaign with the goal of increasing the percentage of patients with STEMI who would receive a primary PCI within 90 minutes of presentation at a PCI-capable hospital to $75 \% .{ }^{19}$ In May 2007, the American Heart Association launched Mission: Lifeline, another national initiative designed to educate patients and providers about the importance of rapid response to STEMI and to help hospitals create coordinated STEMI diagnostic and treatment systems. ${ }^{20}$ 
Table 3 Association between various prognostic factors and failure to receive a primary $\mathrm{PCl}$ within 90 minutes among patients hospitalized with STEMI in 200 I-20I I: Worcester Heart Attack Study

\begin{tabular}{|c|c|c|}
\hline \multirow[t]{2}{*}{ Factors } & $\begin{array}{l}\text { Adjusted for } \\
\text { sociodemographics, } \\
\text { comorbidities, prior } \\
\text { coronary } \\
\text { revascularization }^{\mathrm{a}}\end{array}$ & $\begin{array}{l}\text { Further } \\
\text { adjusted for } \\
\text { in-hospital } \\
\text { factors }^{b}\end{array}$ \\
\hline & \multicolumn{2}{|l|}{ Adjusted RR (95\% Cl) } \\
\hline \multicolumn{3}{|l|}{ Age, years } \\
\hline$<55$ & 1.00 & 1.00 \\
\hline $55-64$ & $0.96(0.69-1.34)$ & $0.93(0.66-1.31)$ \\
\hline $65-74$ & $1.13(0.78-1.64)$ & I.II (0.76-I.63) \\
\hline$\geq 75$ & $1.25(0.86-1.80)$ & I.25 (0.85-I.83) \\
\hline Female (vs male) & $1.14(0.86-1.51)$ & $1.16(0.87-1.55)$ \\
\hline White (vs non-White) & $0.82(0.53-1.28)$ & $0.81(0.51-1.27)$ \\
\hline Married (vs unmarried) & $1.00(0.77-1.28)$ & I.0I (0.78-I.3I) \\
\hline \multicolumn{3}{|l|}{ Comorbid conditions } \\
\hline Angina & I.I9 (0.8I-1.74) & $1.28(0.86-1.90)$ \\
\hline Atrial fibrillation & $1.22(0.7 I-2.09)$ & I. I $8(0.69-2.04)$ \\
\hline Chronic kidney disease & $1.12(0.74-1.70)$ & $1.12(0.73-1.72)$ \\
\hline \multicolumn{3}{|l|}{ pulmonary disease } \\
\hline Depression & 0.91 (0.62-1.32) & $0.94(0.64-1.38)$ \\
\hline Diabetes & $1.26(0.95-1.66)$ & $1.28(0.96-1.70)$ \\
\hline Heart failure & $0.97(0.58-1.63)$ & $0.99(0.58-I .7 I)$ \\
\hline Hypertension & $0.82(0.63-1.07)$ & $0.82(0.63-1.07)$ \\
\hline $\begin{array}{l}\text { Peripheral vascular } \\
\text { disease }\end{array}$ & $0.97(0.66-1.42)$ & $0.98(0.66-1.46)$ \\
\hline Stroke & $0.83(0.44-1.54)$ & $0.88(0.47-1.64)$ \\
\hline Prior PCl & $0.96(0.70-1.31)$ & $0.90(0.63-1.30)$ \\
\hline Prior CABG surgery & $1.94(1.20-3.14)$ & $1.88(1.13-3.12)$ \\
\hline Initial AMI (vs prior AMI) & & $0.88(0.6 \mathrm{I}-1.25)$ \\
\hline $\begin{array}{l}\text { Arrival at ED during off-hours } \\
\text { (vs regular hours) }\end{array}$ & & $1.44(1.13-1.85)$ \\
\hline $\begin{array}{l}\text { Arrival at ED by carl } \\
\text { walked-in (vs ambulance) }\end{array}$ & & $1.57(1.20-2.07)$ \\
\hline \multicolumn{3}{|c|}{$\begin{array}{l}\text { Notes: }{ }^{2} \text { Adjusted for study period, sociodemographic characteristics, previously } \\
\text { diagnosed comorbid conditions, and prior coronary revascularization (PCl or CABG } \\
\text { surgery). }{ }^{\circ} \text { Adjusted for study period, sociodemographic characteristics, previously } \\
\text { diagnosed comorbid conditions, prior coronary revascularization (PCl or CABG } \\
\text { surgery), AMl order, ED arrival time, and mode of transportation. }\end{array}$} \\
\hline
\end{tabular}

Several studies in the US have shown reductions in door-to-balloon times since these national efforts have been employed. ${ }^{4-6}$ Findings from the Blue Cross Blue Shield of Michigan Cardiovascular Consortium of 8,771 patients with STEMI who underwent a primary PCI at nine hospitals between 2003 and 2008 showed that the median door-toballoon time had decreased from 113 minutes in 2003 to 76 minutes in $2008(P<0.001)$. In addition, the percentage of patients who were revascularized with a door-to-balloon time of $<90$ minutes significantly increased from $29 \%$ in
Table 4 Association between various prognostic factors and failure to receive a primary $\mathrm{PCl}$ within 90 minutes among patients hospitalized with STEMI in 2001/2003 and 2009/201 I: Worcester Heart Attack Study

\begin{tabular}{|c|c|c|}
\hline \multirow[t]{2}{*}{ Factors } & $2001 / 2003$ & 2009/20II \\
\hline & \multicolumn{2}{|l|}{$\begin{array}{l}\text { Adjusted RR } \\
(95 \% \mathrm{Cl})^{\mathrm{a}}\end{array}$} \\
\hline \multicolumn{3}{|l|}{ Age, years } \\
\hline$<55$ & 1.00 & 1.00 \\
\hline $55-64$ & I.0I (0.58-I.77) & $1.55(0.77-3.13)$ \\
\hline $65-74$ & $1.23(0.60-2.49)$ & $\mathrm{I} .07(0.50-2.30)$ \\
\hline$\geq 75$ & I. $18(0.60-2.29)$ & $1.23(0.57-2.66)$ \\
\hline Female (vs male) & $1.13(0.70-1.83)$ & $1.55(0.90-2.68)$ \\
\hline White (vs non-White) & $0.93(0.29-2.94)$ & $0.45(0.19-1.05)$ \\
\hline Married (vs unmarried) & $1.01(0.65-1.57)$ & $0.88(0.53-1.45)$ \\
\hline \multicolumn{3}{|l|}{ Comorbid conditions } \\
\hline Angina & $1.40(0.79-2.49)$ & $5.52(1.62-18.79)$ \\
\hline Atrial fibrillation & $0.81(0.30-2.22)$ & $1.20(0.31-4.57)$ \\
\hline Chronic kidney disease & $0.96(0.44-2.09)$ & $0.43(0.14-1.34)$ \\
\hline $\begin{array}{l}\text { Chronic obstructive } \\
\text { pulmonary disease }\end{array}$ & $1.01(0.55-1.83)$ & $1.12(0.47-2.69)$ \\
\hline Depression & $1.10(0.53-2.26)$ & $0.85(0.42-1.72)$ \\
\hline Diabetes & $0.94(0.57-1.53)$ & $1.72(1.01-2.91)$ \\
\hline Heart failure & $1.16(0.39-3.45)$ & $0.25(0.05-1.17)$ \\
\hline Hypertension & $0.89(0.58-1.37)$ & $0.71(0.39-1.29)$ \\
\hline $\begin{array}{l}\text { Peripheral vascular } \\
\text { disease }\end{array}$ & $0.90(0.32-2.5 I)$ & $1.01(0.46-2.19)$ \\
\hline Stroke & $0.96(0.40-2.33)$ & $0.53(0.07-4.27)$ \\
\hline Prior $\mathrm{PCl}$ & $0.73(0.37-1.40)$ & $0.74(0.30-1.82)$ \\
\hline Prior CABG surgery & $1.17(0.36-3.77)$ & $2.70(1.13-6.47)$ \\
\hline Initial AMI (vs prior AMI) & $0.86(0.49-1.48)$ & $0.61(0.24-1.60)$ \\
\hline $\begin{array}{l}\text { Arrival at ED during } \\
\text { off-hours (vs regular hours) }\end{array}$ & $1.38(0.92-2.08)$ & $1.42(0.86-2.35)$ \\
\hline $\begin{array}{l}\text { Arrival at ED by car/ } \\
\text { walked-in (vs ambulance) }\end{array}$ & I.2I (0.7I-2.05) & $1.63(0.97-2.73)$ \\
\hline
\end{tabular}

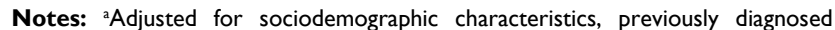
comorbid conditions, prior coronary revascularization (PCl or CABG surgery), AMI order, ED arrival time, and mode of transportation.

Abbreviations: $\mathrm{PCl}$, percutaneous coronary intervention; STEMI, ST-segment elevation myocardial infarction; RR, risk ratio; CABG, coronary artery bypass graft; $\mathrm{AMl}$, acute myocardial infarction; $\mathrm{ED}$, emergency department; $\mathrm{Cl}$, confidence interval.

2003 to $67 \%$ in $2008(P<0.001) .{ }^{4}$ A prior study that examined data from $>300,000$ Medicare patients at 900 US hospitals found that door-to-balloon times declined from a median of 96 minutes in 2005 to 64 minutes in 2010. There were corresponding increases in the percentage of patients who had door-to-balloon times $<90$ minutes $(44 \%-91 \%){ }^{5} \mathrm{~A}$ recent study analyzed data from 96,738 admissions for patients with STEMI who underwent a primary PCI from July, 2005, through June, 2009, at 515 US hospitals participating in the CathPCI Registry. Median door-to-balloon times declined significantly from 83 minutes in the first year to 67 minutes in the most recent study year, and the percentage of patients for whom the door-to-balloon time was 90 minutes or less increased from $60 \%$ to $83 \%$ during the years under study 
$(P<0.001) .{ }^{6}$ Consistent with the timeline of national efforts in reducing door-to-balloon time and prior research results, our current decade-long trend study observed a substantial decrease in the median door-to-balloon time and a dramatic increase in the proportion of patients who received a primary PCI within the guideline-recommended 90 minutes since 2005/2007. Indeed, two studies have been previously published from our institution, which examined the positive impact of a regional system in central Massachusetts for STEMI care on shortening the door-to-balloon times for these patients during the past several years. ${ }^{21,22}$

While reductions in door-to-balloon times have taken place in many US hospitals over time, some unintended consequences of these efforts merit attention. Corresponding to the national effort initiated by the American College of Cardiology in 2006 to reduce door-to-balloon times, several strategies and organizational factors associated with shorter door-to-balloon time have been identified and promoted. ${ }^{23-25}$ These efforts include encouraging emergency medical service (EMS) providers and ED physicians to activate the cardiac catheterization laboratory prior to consultation with a staff cardiologist, which may have achieved a significant reduction in door-to-balloon time, while increasing the rate of "false activations". Indeed, a prior study of all adult patients with a suspected STEMI between 2007 and 2011 at the University of Michigan Hospital noted that the median door-to-balloon time decreased from 67 minutes in 2007 to 55 minutes in 2011 , but the false activation rates increased from $15 \%$ to $40 \%$ of all cases. ${ }^{26}$ When the cardiac catheterization laboratory is activated emergently, resources must be collected to prepare for a potential patient. During off-hours, this often requires bringing in a full team to begin preparing the cardiac catheterization laboratory. These false cardiac catheterization laboratory activations can be a drain on staff and a poor use of resources. Therefore, future studies of health care system interventions to decrease the rates of false cardiac catheterization laboratory activations while maintaining short door-to-balloon times remain warranted.

\section{Factors associated with failure to receive a primary $\mathrm{PCl}$ within 90 minutes after ED arrival}

A recent systematic review and meta-analysis examining the association between off-hour presentation and outcomes in patients with AMI has suggested that patients with STEMI presenting during off-hours have longer door-to-balloon times. ${ }^{27}$ A prior study examined data from the Get With the Guidelines-Coronary Artery Disease databases between
2000 and 2005 and found that, among the 5,454 patients with STEMI who received a primary PCI, those arriving during off-hours were less likely to achieve door-to-balloon times $\leq 90$ minutes compared with those arriving during regular hours. ${ }^{28}$ In the National Registry of Myocardial Infarction study of 33,647 patients with STEMI treated with primary PCI from 1999 to $2002,{ }^{15} 54 \%$ of patients were treated during off-hours; door-to-balloon times were substantially longer during off-hours (116 minutes) than regular hours (95 minutes). Longer door-to-balloon times during off-hours were primarily due to a longer interval between obtaining the electrocardiogram (ECG) and patient arrival at the cardiac catheterization laboratory. Similarly, our study noted that patients with STEMI admitted to the ED during off-hours were less likely to have received a timely primary PCI compared with those admitted during regular hours. Approaches to provide onsite staffing of the cardiac catheterization laboratory and rapid access to interventional cardiologists during off-hours, including consideration of the costs of providing such coverage, would be beneficial.

In this investigation, we found that patients with STEMI who arrived at the ED by car/walked-in were less likely to have received a primary PCI within 90 minutes, compared with those who arrived by ambulance. Prehospital ECGs have been recommended and are increasingly used in the management of patients with chest pain transported by EMSs, ${ }^{1}$ such that paramedics can rapidly diagnose and triage patients with a suspected STEMI before hospital arrival. Since hospitals can use the prehospital ECG results to activate the cardiac catheterization laboratory while the patient is en route to the hospital, door-to-balloon times are shorter than when activation is initiated after the patient's arrival at the ED. Several studies have shown that the use of prehospital ECGs is associated with shorter door-to-balloon times. ${ }^{29,30}$ Patients who arrive at the ED by private vehicle or other means might be triaged to a lower level of estimated illness severity and, as such, experience delays in the receipt of an ECG and diagnosis of STEMI. Similarly, patients who present with chest pain and arrive at the hospital by EMS may receive more rapid and definitive care due to the importance placed on EMS arrival by providers and perception that patients transported by EMS may be "sicker" than those who arrive by other means.

Although our study also identified that having previously undergone $\mathrm{CABG}$ surgery was significantly associated with not receiving a primary PCI within the guideline-recommended time frame, a prior systematic review of factors associated with door-to-balloon time in patients with STEMI 
treated with PCI has found mixed findings with regard to the strength of association of these factors between studies. ${ }^{31}$ While these differences in study results may be due to differences in study design, definitions of key covariates, patient populations under study, and sample size considerations, our study identified several patient groups at high risk for failing to be treated in a timely manner in whom further surveillance and hospital or provider educational efforts might be directed.

Although several studies, including ours, have suggested encouraging reductions in door-to-balloon times over the years, health care providers should continue their efforts to educate patients about the symptoms of AMI and importance of calling 911 to facilitate EMS triage, treatment, and transport to reduce not only in-hospital but also prehospital treatment delays. Indeed, delays in patient's medical care seeking behavior following the development of acute coronary symptoms continue to remain unduly long and have improved little over time. ${ }^{32,33}$

\section{Strengths and limitations of the study}

The strengths of the present community-based study include the examination of relatively contemporary decade-long trends in, and factors associated with, door-to-balloon time among patients hospitalized with STEMI. However, several limitations need to be acknowledged in the interpretation of the present findings. Since our study population included only patients who had received a primary PCI at the two major PCI-capable urban teaching and community hospitals in central Massachusetts, one needs to be careful in extrapolating our findings to those who reside in other geographic areas. Because study patients were predominantly White, the generalizability of our findings to other race/ethnic groups may be limited. In addition, there remains the potential for unmeasured confounding in our observed associations since we did not have information available on several patientassociated characteristics, such as education, psychosocial factors, and treatment preference, as well as detailed health care system-level factors, which may have affected door-toballoon times. Since our study was restricted to patients who were hospitalized with STEMI and received a primary PCI at a PCI-capable hospital in central Massachusetts between 2001 and 2011, we aggregated data from the six individual study years in order to increase the overall sample size. Therefore, our subgroup analysis using data from the first and the last 2-year time clusters was limited in providing statistically stable results.

\section{Conclusion}

Between 2001 and 2011, the likelihood of receiving a primary PCI within guideline-recommended times among patients who were hospitalized with STEMI at the two major teaching and community hospitals in central Massachusetts has increased dramatically. Although most of the identified risk factors for the less than optimal timely receipt of a primary PCI were not modifiable, our findings can hopefully lead to better development of innovative, patient-centered, intervention strategies, which can further reduce the door-to-balloon times of patients hospitalized with STEMI and enhance their hospital and postdischarge outcomes.

\section{Acknowledgments}

We are indebted to the physicians and nurses who have worked on the Worcester Heart Attack Study for the past several decades. This research was made possible by the cooperation of participating hospitals in the Worcester metropolitan area. Funding support was provided by the National Institutes of Health (RO1 HL35434).

\section{Disclosure}

Partial salary support for JM Gore and RJ Goldberg was provided by the National Institutes of Health grant 1U01HL105268-01. The authors report no other conflicts of interest in this work.

\section{References}

1. O'Gara PT, Kushner FG, Ascheim DD, et al. American College of Cardiology Foundation/American Heart Association Task Force on Practice Guidelines. 2013 ACCF/AHA guideline for the management of ST-elevation myocardial infarction: a report of the American College of Cardiology Foundation/American Heart Association Task Force on Practice Guidelines. J Am Coll Cardiol. 2013;61(4):e78-e140.

2. Gershlick AH, Banning AP, Myat A, Verheugt FW, Gersh BJ. Reperfusion therapy for STEMI: is there still a role for thrombolysis in the era of primary percutaneous coronary intervention? Lancet. 2013;382(9892):624-632.

3. McNamara RL, Herrin J, Bradley EH, et al. Hospital improvement in time to reperfusion in patients with acute myocardial infarction, 1999 to 2002. J Am Coll Cardiol. 2006;47(1):45-51.

4. Flynn A, Moscucci M, Share D, et al. Trends in door-to-balloon time and mortality in patients with ST-elevation myocardial infarction undergoing primary percutaneous coronary intervention. Arch Intern Med. 2010;170(20):1842-1849.

5. Krumholz HM, Herrin J, Miller LE, et al. Improvements in doorto-balloon time in the United States, 2005 to 2010. Circulation. 2011;124(9):1038-1045.

6. Menees DS, Peterson ED, Wang Y, et al. Door-to-balloon time and mortality among patients undergoing primary PCI. $N$ Engl J Med. 2013;369(10):901-909.

7. Goldberg RJ, Gore JM, Alpert JS, Dalen JE. Recent changes in attack and survival rates of acute myocardial infarction (1975 through 1981) the Worcester Heart Attack Study. JAMA. 1986;255(20):2774-2779. 
8. Goldberg RJ, Gore JM, Alpert JS, Dalen JE. Incidence and case fatality rates of acute myocardial infarction (1975-1984): the Worcester Heart Attack Study. Am Heart J. 1988;115(4):761-767.

9. Goldberg RJ, Yarzebski J, Lessard D, Gore JM. A two-decades (1975 to 1995) long experience in the incidence, in-hospital and long-term case-fatality rates of acute myocardial infarction: a community-wide perspective. J Am Coll Cardiol. 1999;33(6):1533-1539.

10. Floyd KC, Yarzebski J, Spencer FA, et al. A 30-year perspective (19752005 ) into the changing landscape of patients hospitalized with initial acute myocardial infarction: Worcester Heart Attack Study. Circ Cardiovasc Qual Outcomes. 2009;2(2):88-95.

11. McManus DD, Gore J, Yarzebski J, Spencer F, Lessard D, Goldberg RJ. Recent trends in the incidence, treatment, and outcomes of patients with STEMI and NSTEMI. Am J Med. 2011;124(1):40-47.

12. Rathore SS, Curtis JP, Chen J, et al. National Cardiovascular Data Registry. Association of door-to-balloon time and mortality in patients admitted to hospital with ST elevation myocardial infarction: national cohort study. BMJ. 2009;338:b1807.

13. Zou G. A modified Poisson regression approach to prospective studies with binary data. Am J Epidemiol. 2004;159(7):702-706.

14. Spencer FA, Montalescot G, Fox KA, et al. Global Registry of Acute Coronary Events (GRACE) Investigators. Delay to reperfusion in patients with acute myocardial infarction presenting to acute care hospitals: an international perspective. Eur Heart J. 2010;31(11):1328-1336.

15. Magid DJ, Wang Y, Herrin J, et al. Relationship between time of day, day of week, timeliness of reperfusion, and in-hospital mortality for patients with acute ST-segment elevation myocardial infarction. JAMA. 2005;294(7):803-812.

16. Ryan TJ, Antman EM, Brooks NH, et al. 1999 update: ACC/AHA guidelines for the management of patients with acute myocardial infarction: executive summary and recommendations: a report of the American College of Cardiology/American Heart Association Task Force on Practice Guidelines (Committee on Management of Acute Myocardial Infarction). Circulation. 1999;100(9):1016-1030.

17. Antman EM, Anbe DT, Armstrong PW, et al. American College of Cardiology/American Heart Association Task Force on Practice Guidelines (Writing Committee to Revise the 1999 Guidelines for the Management of Patients With Acute Myocardial Infarction). ACC/ AHA guidelines for the management of patients with ST-elevation myocardial infarction - executive summary: a report of the American College of Cardiology/American Heart Association Task Force on Practice Guidelines (writing committee to revise the 1999 guidelines for the management of patients with acute myocardial infarction). Circulation. 2004;110(5):588-636.

18. Williams SC, Schmaltz SP, Morton DJ, Koss RG, Loeb JM. Quality of care in U.S. hospitals as reflected by standardized measures, 2002-2004. N Engl J Med. 2005;353(3):255-264.

19. Krumholz HM, Bradley EH, Nallamothu BK, et al. A campaign to improve the timeliness of primary percutaneous coronary intervention. Door-to-balloon: an alliance for quality. JACC Cardiovasc Interv. 2008;1(1):97-104
20. Bagai A, Al-Khalidi HR, Sherwood MW, et al. Regional systems of care demonstration project: Mission: Lifeline STEMI Systems Accelerator: design and methodology. Am Heart J. 2014;167(1):15-21.

21. Cyr J, Paige P, Paige P, Fisher D. Sustaining and spreading reduced door-to-balloon times for ST-segment elevation myocardial infarction patients. Jt Comm J Qual Patient Saf. 2009;35(6):297-306.

22. Darling CE, Smith CS, Sun JE, et al. Cost reductions associated with a quality improvement initiative for patients with ST-elevation myocardial infarction. Jt Comm J Qual Patient Saf. 2013;39(1):16-21.

23. Bradley EH, Herrin J, Wang Y, et al. Door-to-drug and door-to-balloon times: where can we improve? Time to reperfusion therapy in patients with ST-segment elevation myocardial infarction (STEMI). Am Heart J. 2006;151(6):1281-1287.

24. Bradley EH, Curry LA, Webster TR, et al. Achieving rapid door-toballoon times: how top hospitals improve complex clinical systems. Circulation. 2006;113(8):1079-1085.

25. Bradley EH, Herrin J, Wang Y, et al. Strategies for reducing the door-to-balloon time in acute myocardial infarction. $N$ Engl J Med. 2006;355(22):2308-2320.

26. Barnes GD, Katz A, Desmond JS, et al. False activation of the cardiac catheterization laboratory for primary PCI. Am J Manag Care. 2013; 19(8):671-675.

27. Sorita A, Ahmed A, Starr SR, et al. Off-hour presentation and outcomes in patients with acute ischemic stroke: a systematic review and metaanalysis. BMJ. 2014;348:f7393.

28. Jneid H, Fonarow GC, Cannon CP, et al. Impact of time of presentation on the care and outcomes of acute myocardial infarction. Circulation. 2008;117(19):2502-2509.

29. Curtis JP, Portnay EL, Wang Y, et al. National Registry of Myocardial Infarction-4. The pre-hospital electrocardiogram and time to reperfusion in patients with acute myocardial infarction, 2000-2002. Findings from the National Registry of Myocardial Infarction-4. J Am Coll Cardiol. 2006;47(8):1544-1552.

30. Diercks DB, Kontos MC, Chen AY, et al. Utilization and impact of pre-hospital electrocardiograms for patients with acute ST-segment elevation myocardial infarction. Data from the NCDR (National Cardiovascular Data Registry) ACTION (Acute Coronary Treatment and Intervention Outcomes Network) Registry. J Am Coll Cardiol. 2009;53(2):161-166.

31. Peterson MC, Syndergaard T, Bowler J, Doxey R. A systematic review of factors predicting door to balloon time in ST-segment elevation myocardial infarction treated with percutaneous intervention. Int $J$ Cardiol. 2012;157(1):8-23.

32. Nguyen HL, Saczynski JS, Gore JM, Goldberg RJ. Age and sex differences in duration of prehospital delay in patients with acute myocardial infarction: a systematic review. Circ Cardiovasc Qual Outcomes. 2010;3(1):82-92.

33. Nguyen HL, Gore JM, Saczynski JS, et al. Age and sex differences and 20-year trends (1986 to 2005) in prehospital delay in patients hospitalized with acute myocardial infarction. Circ Cardiovasc Qual Outcomes. 2010;3(6):590-598.
Clinical Epidemiology

\section{Publish your work in this journal}

Clinical Epidemiology is an international, peer-reviewed, open access, online journal focusing on disease and drug epidemiology, identification of risk factors and screening procedures to develop optimal preventative initiatives and programs. Specific topics include: diagnosis, prognosis, treatment, screening, prevention, risk factor modification,

Submit your manuscript here: https://www.dovepress.com/clinical-epidemiology-journal
Dovepress

systematic reviews, risk and safety of medical interventions, epidemiology and biostatistical methods, and evaluation of guidelines, translational medicine, health policies and economic evaluations. The manuscript management system is completely online and includes a very quick and fair peer-review system, which is all easy to use. 